\title{
Rapid diagnosis of primary ciliary dyskinesia: cell culture and soft computing analysis
}

\author{
Massimo Pifferi*, Andrew Bush", Francesca Montemurro", Giovanni Pioggia+, \\ Martina Piras*, Gennaro Tartarisco ${ }^{+}$, Maria Di Cicco*, Iolanda Chinellato ${ }^{\$}$, \\ Angela M. Cangiotti ${ }^{f}$ and Attilio L. Boner ${ }^{\S}$
}

ABSTRACT: Diagnosis of primary ciliary dyskinesia (PCD) sometimes requires repeated nasal brushing to exclude secondary ciliary alterations. Our aim was to evaluate whether the use of a new method of nasal epithelial cell culture can speed PCD diagnosis in doubtful cases and to identify which are the most informative parameters by means of a multilayer artificial neural network (ANN).

A cross-sectional study was performed in patients with suspected PCD. All patients underwent nasal brushing for ciliary motion analysis, ultrastructural assessment and evaluation of ciliary function after ciliogenesis in culture by ANN.

151 subjects were studied. A diagnostic suspension cell culture was obtained in 117 nasal brushings. A diagnosis of PCD was made in 36 subjects (29 of whom were children). In nine out of the 36 patients the diagnosis was made only after a second brushing, because of equivocal results of both tests at first examination. In each of these subjects diagnosis of PCD was confirmed by cell culture results.

Cell culture in suspension evaluated by means of ANN allows the separation of PCD from secondary ciliary dyskinesia patients after only 5 days of culture and allows diagnosis to be reached in doubtful cases, thus avoiding the necessity of a second sample.

KEYWORDS: Ciliary motion analysis, mathematical evaluation, suspension cell culture, transmission electron microscopy

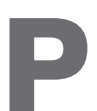

rimary ciliary dyskinesia (PCD), a rare inherited disorder characterised by chronic infection of the upper and lower airway [1, 2], is usually diagnosed by the combination of clinical features together with distinctive transmission electron microscopy (TEM) images and beat frequency and pattern of ciliary motion analysis (CMA) from a nasal brush biopsy [3]. In the relatively few patients in whom these tests are equivocal, there are other tests available, including ciliary culture, immunofluorescence of ciliary proteins and genetic testing [4]. The occasional need for more sophisticated tests is related to the fact that the diagnosis of PCD may be difficult because of respiratory epithelial damage secondary to infection or inflammation. Furthermore, ultrastructural findings may be normal despite alterations in ciliary beat patterns strongly suggestive of a primary disease [5]. It is in these patients that ancillary testing may be particularly valuable. If secondary damage is suspected, a second sample is usually recommended after a period of antibiotic and anti-inflammatory therapy, but even with this strategy, a second brushing may not be diagnostic in $\geqslant 5 \%$ of cases [1]. Alternatively, when CMA and TEM provide equivocal findings, PCD diagnosis can be reached with the culture of ciliated cells since all secondary damage is virtually absent after ciliogenesis in culture. Successful cultures are associated with the ciliogenesis of human respiratory epithelium, which takes place after $24-48 \mathrm{~h}$ of culture when aggregated cells are floating through the medium in the form of spheroids. These consist of a layer of respiratory epithelial cells which maintain cell polarity and, in subjects without $\mathrm{PCD}$, exhibit normal beating cilia at their outer surface (online supplementary video clip 1 ).
AFFILIATIONS

*Dept of Paediatrics, University Hospital of Pisa, Pisa,

"Interdepartmental Research Centre "E. Piaggio", Faculty of Engineering, University of Pisa, Pisa,

${ }^{+}$Institute of Clinical Physiology, National Research Council, Pisa, ${ }^{\S}$ Dept of Paediatrics, University of Verona, Verona, and

IInstitute of Normal Human

Morphology, Electron Microscopy Unit, University Hospital of Ancona, Ancona, Italy.

\#Imperial School of Medicine at the National Heart and Lung Institute,

London, UK.

\section{CORRESPONDENCE}

M. Pifferi

Dept of Paediatrics

University of Pisa

Via Roma 67

56126 Pisa

Italy

E-mail: m.pifferi@med.unipi.it

Received:

March 042012

Accepted after revision:

Aug 022012

First published online:

Aug 162012 
If a frankly abnormal pattern of ciliary movement persists after culture the diagnosis is PCD [6]. In the past, culture was mainly performed on cells obtained by nasal forceps biopsy [6], which is painful. Recently, we have demonstrated that cell culture is possible directly from the brushed nasal epithelial cells, thus reducing the need for a second and more traumatic sampling [7]. With our simplified culture system, the evaluation of ciliary activity is only possible on the 21st day of suspension culture, when new cilia formation has definitely occurred. However, because of the limited amount of material, we are not able to perform electron microscopy, one of the key diagnostic tests in PCD, and this is a weakness of the technique. We therefore hypothesised that, using soft computing analysis of functional parameters in our simplified culture system, we could accurately diagnose PCD, even without structural analysis, which is only available in our unit (Dept of Paediatrics, University Hospital of Pisa, Pisa, Italy) after a mean time of 2 months. Soft computing-based models [8] use methodologies such as multi-layer artificial neural networks (ANNs), which try to mimic the human mind, instructing the computer to deal with imprecision, uncertainty and partial truth, and to include all data in arithmetical algorithms able to counterbalance precision and uncertainty in a way similar to the human brain [8]. They can analyse complex medical data, exploiting meaningful relationships to help physicians in diagnosis, treatment and prediction of clinical outcomes.

Hence, the first aim of this study was to determine whether a precise evaluation of ciliary activity in suspension culture could by itself lead to a firm diagnosis of PCD in a shorter time, using brushed nasal epithelial cells. The secondary aim of the study was to identify which parameters of ciliary activity assessment were most useful for the definitive diagnosis. We assessed, every 5 days, different ciliary activity parameters in cultures, then, with the help of soft computing analysis, we evaluated the whether we could distinguish patients with a final diagnosis of PCD from subjects without PCD.

\section{MATERIALS AND METHODS Subjects}

All the patients with a clinical history and signs suggestive of PCD [2], such as recurrent upper and lower respiratory tract infections and/or presence of situs inversus, sinusitis and/or bronchiectasis, were consecutively evaluated between January 2009 and January 2011 in the Dept of Paediatrics of the University of Pisa. In all subjects, CMA, ultrastructural assessment of cilia and ciliary function after ciliogenesis in culture of nasal brushing samples were performed, according to standard methodologies, when the subject had been free from respiratory infection for $\geqslant 4$ weeks [9]. Ciliary cultures were performed in all subjects on the first occasion. Thus, the sequence for the study was brushing and CMA, and samples sent both for TEM and culture (fig. 1). The differential diagnosis between PCD and secondary ciliary dyskinesia (SCD) was made before TEM was available by means of CMA on nasal brushings and ciliogenesis in culture (i.e. not using ANN data). When TEM became available, it was used as a confirmatory test to differentiate between PCD and SCD.

Definite diagnosis of PCD was made on the basis of structural and/or functional ciliary abnormalities on both brushing and culture, which take account of both beat frequency and pattern,

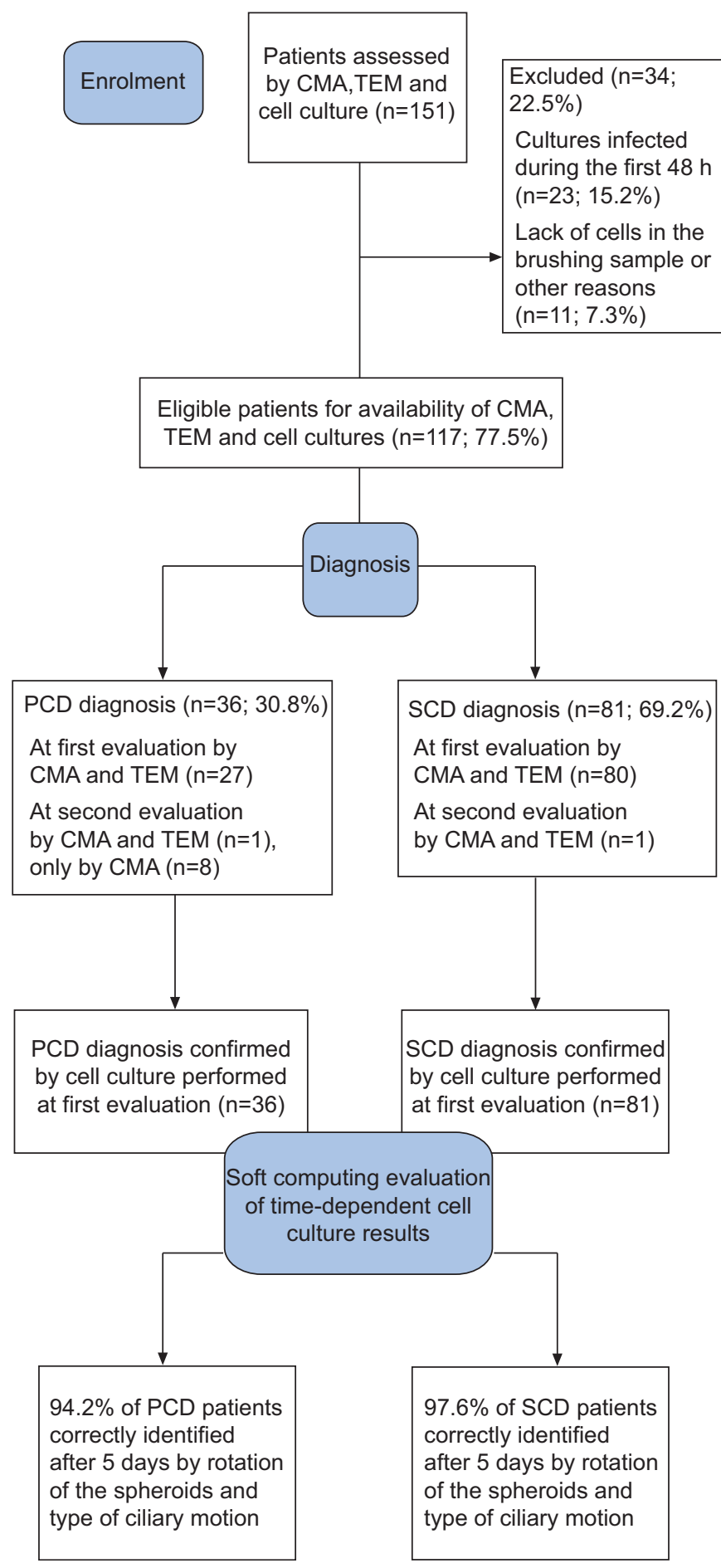

FIGURE 1. Flow diagram of the studied patients. CMA: ciliary motion analysis: TEM: transmission electron microscopy; PCD: primary ciliary dyskinesia; SCD: secondary ciliary dyskinesia.

as previously described [7]. Diagnosis of SCD was made when PCD was excluded and ciliary abnormalities were considered as acquired. In subjects with inconclusive results from the first evaluation with CMA and TEM, the tests were repeated about 4 months later, following appropriate antibiotic and antiinflammatory treatment. From the outset, only one investigator 
(F. Montemurro), who was not involved during the study in any clinical decision-making, was aware of the cell culture results, which would be used for the ANN determinations. At the end of the study and before soft computing evaluation, CMA was also performed by the principal investigator (M. Pifferi). Informed consent for nasal brushing was obtained from each adult patient and from the children's parents. The local Hospital Ethical Committee (University Hospital of Pisa) approved the study protocol.

\section{Cell culture in suspension}

Cell culture was performed as previously reported [7]. Briefly, ciliogenesis of human respiratory epithelium takes place when aggregated cells are floating through the medium in the form of spheroids. Spheroids were identified after $24-48 \mathrm{~h}$ of culture using inverse microscopy (Inverted Microscope TE2000-S; Nikon, Tokyo, Japan) at $\times 60$ and the evaluation of ciliary activity was performed every 5 days until the 20th day of culture. Diagnosis was based on observation of five parameters, considered to be the expression of coordinated ciliary activity, i.e.: 1) rotation of the spheroids (yes or no); 2) migration of the spheroids (yes or no); 3) ability of cilia to remove debris or red blood cells (yes or no); 4) normal motion type; and 5) pathological motion type if found in $\geqslant 40 \%$ of observation fields. A normal ciliary beat pattern or, by contrast, a pathological pattern, such as a reduced ciliary beat frequency with a markedly restricted beat amplitude and a stiff ciliary motion pattern [10], or an abnormal nonflexible beating pattern with a hyperkinetic beat [11], could be easily identified. These parameters were evaluated every 5 days in relation to the final diagnosis as a binary variable: 0 : absence, 1 : presence and evaluated by ANN.

\section{Statistical analyses and soft computing evaluation of cell culture results}

Baseline variables were expressed as group mean \pm SD or as median and interquartile range (IQR) when the variables were non-normally distributed. The diagnosis obtained at the end of the usual diagnostic pathway by ciliary motion analysis, TEM evaluation and based on ciliary activity evaluation after ciliogenesis in culture was accepted as the gold standard to evaluate the different parameters of cell culture suspension as previously reported [7].

The sensitivity and specificity in the diagnosis of PCD of parameters used for ciliary activity evaluation of suspension cell culture and their positive and negative predictive values (PPV and NPV, respectively) were generated using standard equations. All statistical calculations were performed using SPSS version 18.0 software for Windows (SPSS, Chicago, IL, USA) for personal computers.

A soft computing modelling approach was applied to the ciliary activity evaluation during ciliogenesis in culture to make a diagnosis of PCD or SCD, prior to the results of TEM becoming available. In this study, a model was identified by means of a multi-layer ANN. This is a digitised model of a biological brain and can detect complex nonlinear relationships between dependent as well as independent variables in data, which the human brain may fail to detect [12]. In order to evaluate the time-dependent diagnostic efficiency of the model to predict the final diagnosis, a cross-validation procedure of the different cell culture features (rotation of the spheroids, migration of the spheroids, ability of cilia to remove debris, normal ciliary beat pattern and pathological ciliary beat pattern) at days 5, 10, 15 and 20 was applied. Further details on soft computing evaluation of cell culture results are available in the online supplementary material.

\section{RESULTS}

151 subjects were studied (fig. 1). A diagnostically useful suspension cell culture was obtained from nasal brushing samples in 117 (77.5\%; 65 males, 91 children, age range 1.5 months to 57.0 years; median age (IQR) 9.30 (12.0) years) out of 151 subjects (all details are shown in online supplementary table S1). In $23(15.2 \%)$ patients the cultures became infected during the first $48 \mathrm{~h}$ and in 11 (7.3\%) subjects the culture was unsuccessful, either because of a lack of cells in the brushing sample, or because no cilia could be found in spheroids throughout the period of culture. Moreover, because only a small sample could be obtained, the duration of cell culture in suspension, usually 20 days, was reduced to 10 and 15 days, respectively, in nine $(7.7 \%)$ and $10(8.5 \%)$ out of 117 patients.

Ciliary motion analysis (abnormal motion patterns, including immotile cilia and/or very low ciliary beat frequency) and TEM evaluation of cilia (alterations of the central pair and deficiencies of the dynein arms, associated with a small proportion of swollen cilia and compound cilia) allowed the eventual definitive diagnosis of PCD in 36 (30.8\%) subjects (29 children). In nine out of the 36 patients, the diagnosis was obtained only after a second brushing because of equivocal results in one subject or the presence of normal ciliary ultrastructure with nonflexible and hyperkinetic ciliary motion pattern in eight subjects at the first examination (all details shown in online supplementary table S2). In each of these nine subjects, the diagnosis of PCD was strongly suggested prior to any TEM results being obtained by the results of cell cultures (table 1) (online supplementary video clip 2).

In the remaining $81(69.2 \%)$ patients (68 children), ciliary motion analysis demonstrated abnormal patterns in a small proportion of cilia, prevalence of thick cilia and low ciliary beat frequency, compatible with SCD (secondary changes were found in one patient only after the second brushing after intensive and prolonged treatment with antibiotics, but SCD was correctly identified from the culture). In these 81 subjects, TEM evaluation clearly showed nonspecific abnormalities compatible with chronic inflammation (prevalence of swollen cilia and compound cilia). In all these patients the diagnosis of SCD was robustly suggested by the prior results of cell cultures.

Moreover, as can be seen in table 1, ciliary activity evaluation, over the whole 20-day period of suspension cell culture, demonstrated a rotation of the spheroids in 64 out of $81(79 \%)$ subjects with final diagnosis of SCD, and only in one $(2.8 \%)$ out of 36 patients with PCD (rotation of one spheroid 10 days after the start of the cell cultures). In this patient, the diagnosis was confirmed by DNAH11 compound heterozygous mutations [11]. Migration of the spheroids was found in 14 (17.3\%) out of 81 subjects with SCD, but in no PCD patients, and an ability of cilia to remove debris in six $(7.4 \%)$ out of 81 subjects with SCD and in none with PCD. When continuous movements of cilia were detected but migration or rotation of the spheroids with an ability of cilia to remove debris were not observed, a normal 
TABLE 1 Ciliary activity parameters evaluated in suspension cell cultures of patients with primary ciliary dyskinesia (PCD) or secondary ciliary dyskinesia (SCD)

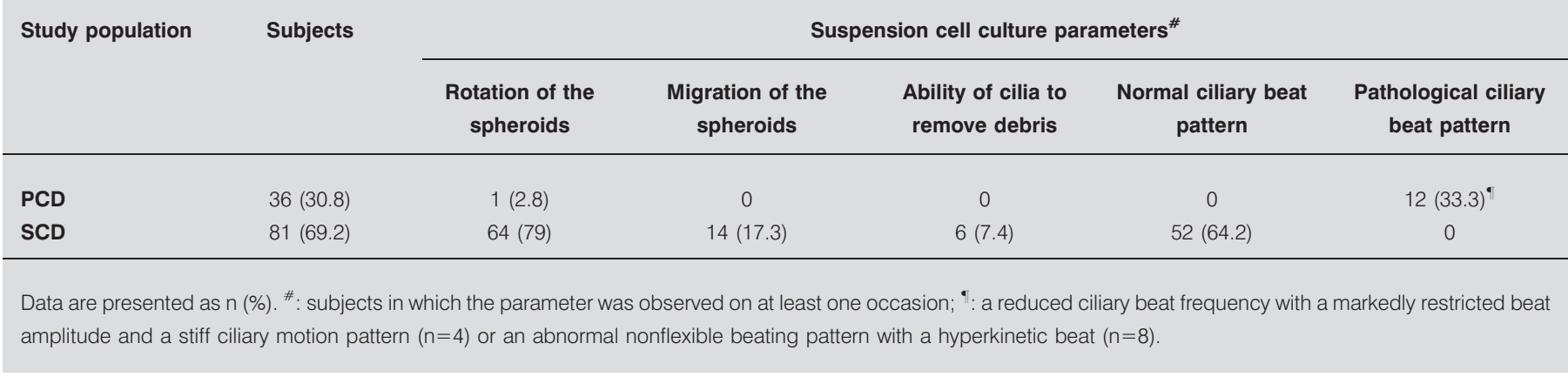

ciliary beat pattern was observed at CMA in $52(64.2 \%)$ out of 81 subjects with SCD, but in no patient with PCD. By contrast, in $12(33.3 \%)$ out of 36 of these PCD patients, but in none with SCD, a pathological ciliary pattern was observed (table 1). In particular, a reduced ciliary beat frequency with a markedly restricted beat amplitude and a stiff ciliary motion pattern or an abnormal nonflexible beating pattern with a hyperkinetic beat were detected in four and eight subjects, respectively.

The sensitivity, specificity, PPVs and NPVs of the different parameters for the diagnosis of PCD are reported in table 2.

Taking into consideration the rotation of the spheroids, migration of the spheroids, ability of cilia to remove debris and type of ciliary motion in the range 5-20 days, the ANNbased model was able to identify the final diagnosis in almost all cases (PCD 99.5 $\pm 0.3 \%$; SCD $98.8 \pm 1.2 \%$ ).

Moreover, since the best combination of sensitivity and specificity was demonstrated by rotation of the spheroids, the ability to correctly distinguish the PCD from SCD by the ANNbased model, taking into account this parameter together with each of the other three, was analysed.

As reported in table 3 , the model was able to correctly identify the PCD or SCD in nearly all cases when the rotation of the spheroids and the type of ciliary motion were considered. This

\begin{tabular}{|c|c|c|c|c|c|}
\hline TABLE 2 & \multicolumn{5}{|c|}{$\begin{array}{l}\text { Sensitivity, specificity, positive predictive values } \\
\text { (PPVs) and negative predictive values (NPVs) of } \\
\text { the different parameters used for ciliary activity } \\
\text { evaluation in suspension cell culture for the } \\
\text { diagnosis of primary ciliary dyskinesia }\end{array}$} \\
\hline \multicolumn{2}{|c|}{$\begin{array}{l}\text { Suspension cell culture } \\
\text { parameters }\end{array}$} & Sensitivity & Specificity & PPV & NPV \\
\hline \multicolumn{2}{|c|}{ Rotation of the spheroids } & 79.0 & 97.2 & 98.5 & 67.3 \\
\hline \multicolumn{2}{|c|}{ Migration of the spheroids } & 17.3 & 100 & 100 & 34.9 \\
\hline \multicolumn{2}{|c|}{$\begin{array}{l}\text { Ability of cilia to remove } \\
\text { debris }\end{array}$} & 7.4 & 100 & 100 & 32.4 \\
\hline \multicolumn{2}{|c|}{ Normal ciliary beat pattern } & 64.2 & 100 & 100 & 55.4 \\
\hline \multicolumn{2}{|c|}{$\begin{array}{l}\text { Pathological ciliary beat } \\
\text { pattern }\end{array}$} & 33.3 & 100 & 100 & 77.1 \\
\hline
\end{tabular}

was true from day 5 of cell culture. Other combinations were less accurate.

\section{DISCUSSION}

The principal finding of the study was that culture in suspension of cells obtained by nasal brushing is possible in around $80 \%$ of the samples, and in our hands, using ANNs, this allowed the differentiation of PCD from SCD within 1 week, and well before TEM results become available.

Strengths of the study include the objective analysis of the data using ANNs, i.e. by mathematical algorithms generated by computer. With this approach, we have been able to identify the two parameters, which, when combined, are most diagnostically accurate (rotation of the spheroids together with type of ciliary motion). These have been used to accurately identify nearly $95 \%$ of PCD patients after only 5 days of culture. ANNs learn from standard data and capture the knowledge contained in the data. Further strengths include the relatively atraumatic nature of the brushing compared to a forceps biopsy. Originally, cells for culture were obtained with more traumatic methods, such as punch biopsy, which may cause pain and bleeding. Punch biopsy also has the disadvantage of requiring a longer time to establish cell culture compared with cells collected by brushing $[7,13,14]$. The technique does, however, have the advantage of a high yield of culture results. Ciliated air-liquid cultures from both punch and brush biopsies [14] result in the culture of sufficient ciliated tissue to allow electron microscopy of cilia, which is not possible with our simplified technique, but the success rate of this technique is only $54 \%$ [14] not $80 \%$ as here. However, with the air-liquid technique, the culture success rate decreases to $26 \%$ in patients with more severe secondary changes, that is, in the very group in whom the risk of a false-positive diagnosis is more likely, and the cells previously had to be cultured for 21 days, increasing the risk of failure [7]. This is exacerbated by the paucity of the starting material, which tends to reduce further in subsequent passages. However, this present study clearly demonstrates that prolonged culture is not necessary to make the diagnosis of PCD.

The major weakness of the study is that TEM is not possible with this technique, but the PPVs of the different ciliary motion patterns resulted in $>98.5 \%$ accuracy, thus allowing the diagnosis in almost all patients. Of course, TEM, when diagnostic (for example, outer dynein arm deficiency), is an important test, but it is not the gold-standard, since cases of PCD with normal ultrastructure are well described $[5,11]$. This was 


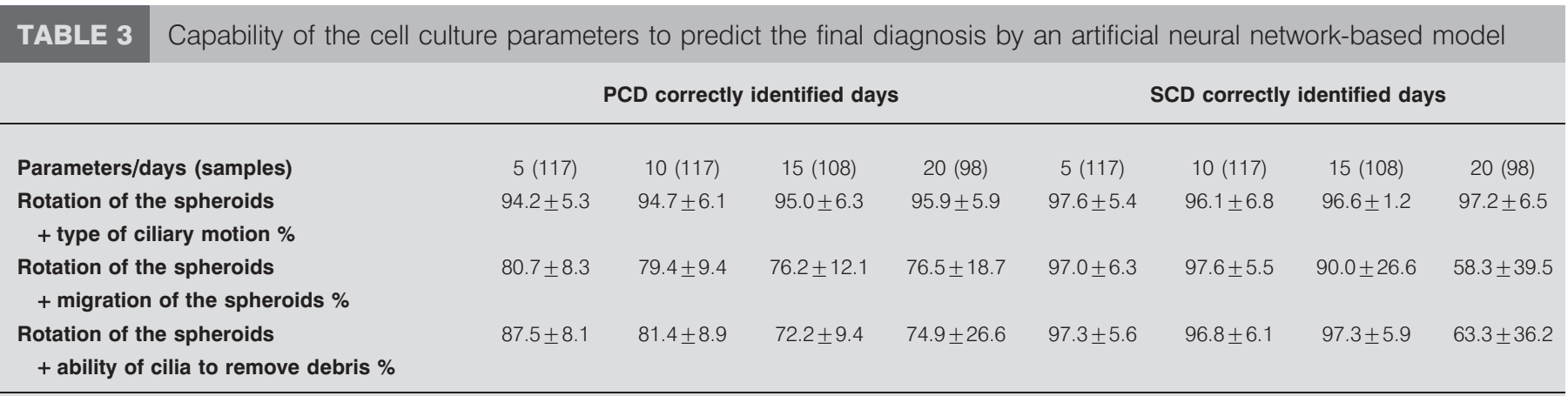

Data are presented as $n$ or mean \pm SD. PCD: primary ciliary dyskinesia; SCD: secondary cliary dyskinesia.

the case in eight of our patients, who were correctly identified from the beginning by the study of ciliogenesis in culture. Even though we cannot perform electron microscopy on cells cultured by our simplified technique, soft computing allowed us to diagnose PCD before the second brushing and without TEM being available in an additional $22 \%$ of the patients within less than a week of the first nasal brushing, without recourse to a second brushing. The result is surprising, given that spheroids of epithelial cells in suspension culture lost cilia during the first week and developed new cilia only after a further 1-2 weeks [15]. We speculate that restoration of normal ciliary activity, in the absence of a congenital defect, does not require the formation of complete new cilia but may occur as a consequence of a normalisation of the normal trafficking of protein complexes between the cell basal body and the cilia tip as required for the assembly, maintenance and function of cilia [16]. In this context, it is worth noting that a second ciliary gene mutation in cis may correct the abnormal phenotype caused by the first, curiously sometimes without apparently restoring the missing substructure [17-20]. We suggest that the integration in a dedicated system of the optical acquisition and the soft computing algorithm will allow the evaluation of time-dependent cell culture results to be gained rapidly, resulting in a cost saving. TEM will thus become a confirmatory tool, given the long delay in obtaining results.

Ideally, we would validate these findings in a second cohort of patients, but it would take a considerable time to generate a sufficiently large number of new patients. However, the network was validated according to a n-fold cross-validation method. Cross-validation is one of several approaches for estimating the performance of a model on unseen data. In a nfold cross-validation, the original dataset is randomly partitioned (split) into $\mathrm{n}$ subsets. For each cross-validation step, a single subset is retained as the validation set (or test set, i.e. validation cohort), and the remaining $n-1$ sub-subsets are used as training set. The cross-validation process is then repeated $n$ times, with each of the $n$ subsets used exactly once as the test set. The $\mathrm{n}$ results from the folds then can be averaged (or otherwise combined) to produce a single estimation. In our work, a five-fold cross-validation process of the different cell culture features at days 5, 10, 15 and 20 was applied; each fold consisted of randomly selected data, and at least one for each category index was included in each fold. We consider that this cross-validation is very robust; much more so than merely splitting the cohort in half using one half as a discovery cohort and one for validation.

The implication of our study is that PCD is rare and difficult to diagnose, and may require repeated testing in doubtful cases $[21,22]$. Problems include, first, that TEM changes may be secondary to environmental mucosal injuries, such as inflammation and bacterial or viral infections, allergies and smoking [23], or may result from inappropriate preparation of a specimen [24]; second, in a few patients with immotile cilia, ciliary ultrastructure may be normal or near normal [25-28]. In this regard, the complexities of determining the gold-standard diagnosis have recently been discussed [4]. We believe this new technique will make diagnosis in doubtful cases significantly easier.

If there is diagnostic doubt, identification of the causative genetic mutations would be ideal, as in our very atypical PCD patient with rotation of spheroids, but PCD is a genetically heterogeneous disease and more than 250 potential candidate genes have been identified [29]. Furthermore, genetic studies have the problem of distinguishing disease-producing mutations from harmless polymorphisms. Other investigations such as nasal nitric oxide are part of the diagnostic process [30], but may be normal in rare patients with PCD and low in subjects with other diseases such as upper airway infections and cystic fibrosis [31]. Under these challenging circumstances, culture of ciliated cells is very helpful, since secondary changes are virtually absent after regrowth of the ciliated epithelium in culture while primary defects remain unchanged [32, 33].

In conclusion, the use of a simple culture technique together with the use of ANNs [34] offers new opportunities for the reliable differentiation of PCD from secondary ciliary dyskinesia.

\section{SUPPORT STATEMENT}

This project was supported by the NIHR Respiratory Disease Biomedical Research Unit at the Royal Brompton and Harefield NHS Foundation Trust and Imperial College London (both London, UK) and by the Fondazione Carlo Laviosa.

\section{STATEMENT OF INTEREST}

None declared.

\section{ACKNOWLEDGEMENTS}

We wish to thank P. Macchia (Dept of Paediatrics, University Hospital of Pisa, Pisa, Italy) for his constant encouragement and support in the 
study of patients with ciliary dyskinesia and for his effort in the organisation of the out- and inpatient clinic for this disease.

\section{REFERENCES}

1 Bush A, Chodhari R, Collins N, et al. Primary ciliary dyskinesia: current state of the art. Arch Dis Child 2007; 92: 1136-1140.

2 Barbato A, Frischer T, Kuehni CE, et al. Primary ciliary dyskinesia: a consensus statement on diagnostic and treatment approaches in children. Eur Respir J 2009; 34: 1264-1276.

3 Meeks M, Bush A. Primary ciliary dyskinesia. Pediatr Pulmonol 2000; 29: 307-316.

4 Hogg C, Bush A. Genotyping in primary ciliary dyskinesia: ready for prime time, or a fringe benefit? Thorax 2012; 67: 377-378.

5 Schwabe GC, Hoffmann K, Loges NT, et al. Primary ciliary dyskinesia associated with normal axoneme ultrastructure is caused by DNAH11 mutations. Hum Mutat 2008; 29: 289-298.

6 Jorissen M, Willems T, Van der Schueren B, et al. Ultrastructural expression of primary ciliary dyskinesia after ciliogenesis in culture. Acta Otorhinolaryngol Belg 2000; 54: 343-356.

7 Pifferi M, Montemurro F, Cangiotti AM, et al. Simplified cell culture method for the diagnosis of atypical primary ciliary dyskinesia. Thorax 2009; 64: 1077-1081.

8 Jamshidi M. Tools for intelligent control: fuzzy controllers, neural networks and genetic algorithms. Philos Transact A Math Phys Eng Sci 2003; 361: 1781-1808.

9 Pifferi M, Cangiotti AM, Ragazzo V, et al. Primary ciliary dyskinesia: diagnosis in children with inconclusive ultrastructural evaluation. Pediatr Allergy Immunol 2001; 12: 274-282.

10 Chilvers MA, Rutman A, O'Callaghan C. Ciliary beat pattern is associated with specific ultrastructural defects in primary ciliary dyskinesia. J Allergy Clin Immunol 2003; 112: 518-524.

11 Pifferi M, Michelucci A, Conidi ME, et al. New DNAH11 mutations in primary ciliary dyskinesia with normal axonemal ultrastructure. Eur Respir J 2010; 35: 1413-1416.

12 Patel JL, Goyal RK. Applications of artificial neural networks in medical science. Curr Clin Pharmacol 2007; 2: 217-226.

13 Dimova S, Brewster ME, Noppe M, et al. The use of human nasal in vitro cell systems during drug discovery and development. Toxicol In Vitro 2005; 19: 107-122.

14 Hirst RA, Rutman A, Williams G, et al. Ciliated air-liquid cultures as an aid to diagnostic testing of primary ciliary dyskinesia. Chest 2010; 138: 1441-1447.

15 Neugebauer P, Endepols H, Mickenhagen A, et al. Ciliogenesis in submersion and suspension cultures of human nasal epithelial cells. Eur Arch Otorhinolaryngol 2003; 260: 325-330.

16 Rosenbaum JL, Witman GB. Intraflagellar transport. Nat Rev Mol Cell Biol 2002; 3: 813-825.
17 Huang B, Ramanis Z, Luck DJ. Suppressor mutations in Chlamydomonas reveal a regulatory mechanism for flagellar function. Cell 1982; 28: 115-124.

18 Porter ME, Knott JA, Gardner LC, et al. Mutations in the SUP-PF-1 locus of Chlamydomonas reinhardtii identify a regulatory domain in the $\beta$-dynein heavy chain. J Cell Biol 1994; 126: 1495-1507.

19 Porter ME, Power J, Dutcher SK. Extragenic suppressors of paralyzed flagellar mutations in Chlamydomonas reinhardtii identify loci that alter the inner dynein arms. J Cell Biol 1992; 118: 1163-1176.

20 Heuser $\mathrm{T}$, Raytchev $\mathrm{M}$, Krell J, et al. The dynein regulatory complex is the nexin link and a major regulatory node in cilia and flagella. J Cell Biol 2009; 187: 921-933.

21 O'Callaghan C, Rutman A, Williams GM, et al. Inner dynein arm defects causing primary ciliary dyskinesia: repeat testing required. Eur Respir J 2011; 38: 603-607.

22 Stannard WA, Chilvers MA, Rutman AR, et al. Diagnostic testing of patients suspected of primary ciliary dyskinesia. Am J Respir Crit Care Med 2010; 181: 307-314.

23 Escudier E, Duquesnoy P, Papon JF, et al. Ciliary defects and genetics of primary ciliary dyskinesia. Paediatr Respir Rev 2009; 10: 51-54.

24 Thomas B, Rutman A, O'Callaghan C. Disrupted ciliated epithelium shows slower ciliary beat frequency and increased dyskinesia. Eur Respir J 2009; 34: 401-404.

25 Greenstone MA, Dewar A, Cole PJ. Ciliary dyskinesia with normal ultrastructure. Thorax 1983; 38: 875-876.

26 Escudier E, Escalier D, Homasson JP, et al. Unexpectedly normal cilia and spermatozoa in an infertile man with Kartagener's syndrome. Eur J Respir Dis 1987; 70: 180-186.

27 Conraads VM, Galdermans DI, Kockx MM, et al. Ultrastructurally normal and motile spermatozoa in a fertile man with Kartagener's syndrome. Chest 1992; 102: 1616-1618.

28 Rutland J, de Iongh RU. Random ciliary orientation. A cause of respiratory tract disease. N Engl J Med 1990; 323: 1681-1684.

29 Zariwala MA, Knowles MR, Omran H. Genetic defects in ciliary structure and function. Annu Rev Physiol 2007; 69: 423-450.

30 Pifferi M, Caramella D, Cangiotti AM, et al. Nasal nitric oxide in atypical primary ciliary dyskinesia. Chest 2007; 131: 870-873.

31 Corbelli R, Hammer J. Measurement of nasal nitric oxide. Paediatr Respir Rev 2007; 8: 269-272.

32 Jorissen M, Willems T, Van der Schueren B, et al. Secondary ciliary dyskinesia is absent after ciliogenesis in culture. Acta Otorhinolaryngol Belg 2000; 54: 333-342.

33 Bukowy Z, Ziętkiewicz E, Witt M. In vitro culturing of ciliary respiratory cells - a model for studies of genetic diseases. J Appl Genet 2011; 52: 39-51.

34 Cleophas TJ, Cleophas TF. Artificial intelligence for diagnostic purposes: principles, procedures and limitations. Clin Chem Lab Med 2010; 48: 159-165. 